\title{
Active Tensile Modulus of an Epithelial Monolayer
}

\author{
Romaric Vincent, ${ }^{*}$ Elsa Bazellières, Carlos Pérez-González, Marina Uroz, and Xavier Serra-Picamal \\ Institute for Bioengineering of Catalonia, Barcelona 08028, Spain \\ Xavier Trepat \\ Institut Català de Recerca i Estudis Avançats (ICREA), Barcelona 08028, Spain \\ and Institute for Bioengineering of Catalonia, Barcelona 08028, Spain
}

(Received 3 March 2015; published 11 December 2015)

\begin{abstract}
A general trait of cell monolayers is their ability to exert contractile stresses on their surroundings. The scaling laws that link such contractile stresses with the size and geometry of constituent cells remain largely unknown. In this Letter, we show that the active tension of an epithelial monolayer scales linearly with the size of the constituent cells, a surprisingly simple relationship. The slope of this relationship defines an active tensile modulus, which depends on the concentration of myosin and spans more than 2 orders of magnitude across cell types and molecular perturbations.
\end{abstract}

Epithelial cell monolayers are the simplest living tissues that exist in nature, yet they are responsible for a complex repertoire of physiological functions. This repertoire includes the ability to self-repair after injury, to adopt complex shapes during morphogenesis, to compartmentalize organs into functional units, and to protect organisms against environmental pathogens [1]. Epithelial monolayers have been the subject of study for more than a century [2], but the advent of life imaging and force microscopy techniques has accelerated our fundamental understanding of their mechanics. Recent discoveries include the ability of cell monolayers to oscillate spontaneously [3,4], to exhibit turbulence and fingering instabilities [5-7], to jam as a function of cell density and geometry [8-10], to build up a surface tension that scales with monolayer size [11], and to display large dynamic heterogeneities [12].

Understanding this diverse and seemingly contradictory phenomenology requires a detailed knowledge of the rheological properties of epithelial monolayers and of their constitutive cells. Over relatively short time scales, these properties have been extensively measured and their origin is known to be passive $[13,14]$. By contrast, over the slow time scales relevant to cell migration, division, and remodeling, the scaling relationships that link monolayer stress, strain, and their rates are unknown. In this slow regime, cells behave as active materials $[15,16]$, the fluctuation dissipation theorem breaks down [17-20], and physical forces trigger mechanotransduction feedback loops that control cytoskeleton remodeling and gene transcription [21,22]. Given the complexity of this scenario, cellular stress and strain would seem unlikely to obey a unique constitutive relationship. Whether a shear modulus can be meaningfully defined over time scales of hours is, thus, unclear.

Here we use 2D epithelial tissues as a model system to ask whether there exists a universal relationship between active tissue tension and cellular strain. We found that monolayer tension varies linearly with strain so as to define an active tensile modulus. This modulus spans more than 2 orders of magnitude across cell types and molecular perturbations, and it depends on the concentration of myosin that can be activated in the cell.

Using polydimethylsiloxane (PDMS) micropatterning techniques as described in Serra-Picamal et al. [4], we seed a (30-50)-cell-wide stripe of confluent cells on top of a soft and elastic polyacrylamide substrate. After removal of the PDMS membrane, the tissue invades the free space mainly by spreading of its constituent cells. We use timelapse microscopy to image monolayer expansion every 3 min during $15 \mathrm{~h}$. To map the velocity components $\left(V_{x}, V_{y}\right)$ of the expanding tissue, we use velocimetry algorithms on the phase contrast images [5]. To map tractions applied by the cells on the deformable polyacrylamide substrate, we use monolayer traction microscopy $[4,23]$.

We study seven different experimental conditions, including five different cell lines [Madin-Darby canine kidney epithelial cells (MDCK), human mammary epithelial cells (MCF10a), human retinal pigment epithelial cells (RPE1), human breast carcinoma cells overexpressing E-cadherin (MDA-Ecad), and human epidermoid carcinoma cells (A431)] and two pharmacological treatments of the MDCK cells, $25 \mu \mathrm{M}$ blebbistatin and $10 \mu \mathrm{M}$ hepatocyte growth factor (HGF). Blebbistatin inhibits myosin contractility, while HGF boosts actin polymerization dynamics and induces scattering of the monolayer.

Figure 1 shows phase contrast images of the seven conditions immediately after removal of the patterning membrane [Figs. 1(a)-1(g)], and after 240 min of expansion [Figs. 1(h)-1(n)]. These images show that the texture of the monolayer is diverse and that the size and aspect ratio of constituent cells varies broadly across cell types and 

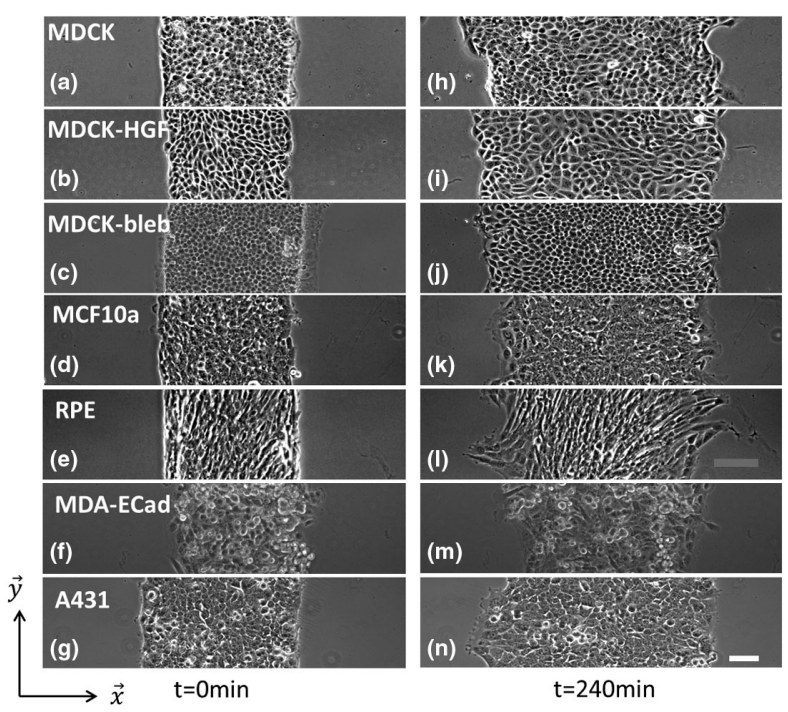

FIG. 1. Phase contrast images of expanding monolayers just after removal of the PDMS membrane (a)-(g) and after $240 \mathrm{~min}$ of expansion (h)-(n) for seven different experimental systems: MDCK (a),(h), MDCK-HGF (b),(i), MDCK-blebbistatin (c),(j), MCF10a (d),(k), RPE1 (e),(l), MDA-Ecad (f),(m), and A431 (g),(n). Scale bar is $100 \mu \mathrm{m}$.

treatments. The organization of the cytoskeleton and the localization and concentration of phosphorylated myosin also display clear differences across cell types (Supplemental Material [24], Figs. S1-S3).

The cell monolayers are designed with a large length-towidth aspect ratio so that the system expands mostly in the horizontal dimension ( $x$ dimension). To ensure that this is the case, we first determine the strain rate tensor from the spatial gradient of the velocity field $\dot{\epsilon}_{i j}=\partial V_{i} / \partial j$. To avoid boundary effects, we average $\dot{\epsilon}_{i j}$ over a region of interest (ROI) defined as the central third of the tissue's width before migration, cutting $10 \%$ of the top and bottom limits of the field of view along the $y$ axis.

We then integrate in time the spatial averages of the strain rate components in order to obtain the strain components $\epsilon_{i j}$, taking care of correcting for the cell division rate [24]. The strain values obtained using this strategy are similar to those obtained by measuring strain directly from image segmentation (Supplemental Material [24] Fig. S4). The evolution in time of $\epsilon_{x x}$ and $\epsilon_{y y}$ clearly shows that in all experimental conditions the monolayer expands dominantly in the $x$ direction (i.e., $\epsilon_{x x} \gg \epsilon_{y y}$ ) (Supplemental Material [24] Fig. S5). In the first hours after removing the PDMS membrane, cells in all monolayers remain cohesive and deform in the $x$ direction at rates that vary more than 1 order of magnitude across cell types and treatments [Fig. 2(a)]. After this initial phase of spreading, monolayers exhibit distinct behaviors. RPE1, MCF10a, MDA-Ecad, and A431 cells, as well as MDCK cells treated with blebbistatin either reach a plateau or tend to it. By contrast, untreated MDCK cells exhibit underdamped
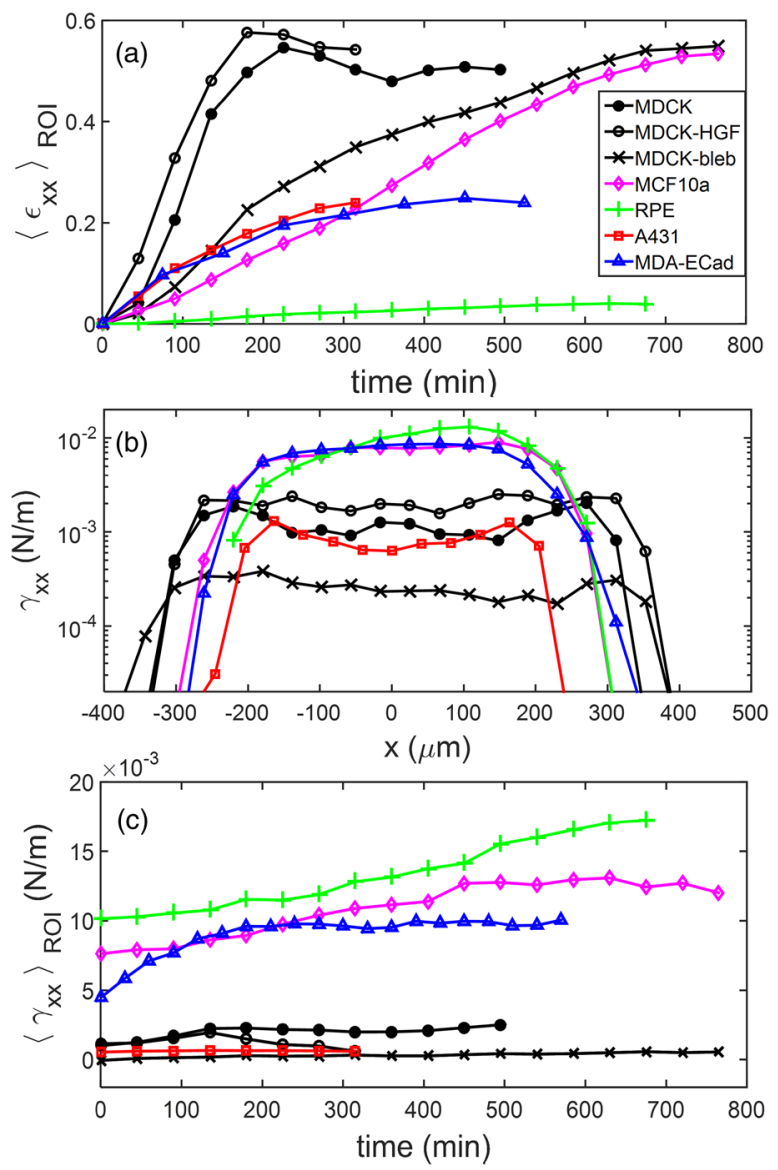

FIG. 2 (color online). (a) Time evolution of the strain $\epsilon_{x x}$ averaged over the central ROI (1/3 of the initial pattern width) for the seven experimental systems. MDCK (black filled circles), MDCK-HGF (black open circles), MDCK-blebbistatin (black crosses), MCF10a (magenta diamonds), RPE (green crosses), MDA-Ecad (blue triangles), and A431 (red squares). Each curve displays a representative experiment of $n \geq 3$ experiments per condition. For each condition we display only the initial phase of expansion. The complete time course for each condition can be found in Supplemental Material [24] Fig. S7 and additional experimental repeats can be found in Supplemental Material [24] Fig. S8. (b) Profile of tension across the monolayer for the seven experimental systems at time $120 \mathrm{~min}$ after the start of the expansion; same symbols as Fig. 2(a). (c) Temporal evolution of the average of tension $\gamma_{x x}$ over the ROI for the seven experimental systems; same symbols as Fig. 2(a).

oscillations as previously described [4], and MDCK cells treated with HGF break cell-cell adhesions and scatter (Supplemental Material [24] Fig. S6).

Taking advantage of the quasiunidimensionality of the system, we use a simple force balance in the $x$ direction to measure monolayer tension $\left(\gamma_{x x}\right)$ [23]. Tension is generated by the actomyosin network and is transmitted through cell-cell adhesions across distances larger than the size of a single cell [12]. $\gamma_{x x}$ is obtained from the spatial integration of the $x$ component of the discrete traction field as 


$$
\gamma_{x x}(i)=\sum_{k=1}^{i}\left\langle T_{x}(k, j)\right\rangle_{j} \Delta x
$$

where $\left\langle T_{x}(k, j)\right\rangle_{j}$ is the $y$-averaged $x$ component of the tractions at coordinate $i$, and $\Delta x$ is the spatial resolution of the traction microscopy setup. $\gamma_{x x}$ has units of surface tension $(\mathrm{N} / \mathrm{m})$.

The obtained tension profiles are plotted in Fig. 2(b) at an experimental time of $120 \mathrm{~min}$. In all experimental conditions, tension builds up monotonically from the edge of the monolayer to reach a plateau in the central region. The height of this plateau varies nearly 2 orders of magnitude between the different conditions. MCF10a, RPE1, and MDA-Ecad monolayers exhibit much higher tension at the monolayer's center than MDCK and A431 cells. As expected, blebbistatin reduces very significantly the amplitude of monolayer tension while the effect of HGF is minor at short times [Fig. 2(b)].

We next focus on the time evolution of average tension in the central region of the tissue, $\left\langle\gamma_{x x}\right\rangle_{\mathrm{ROI}}$. In all monolayers, $\left\langle\gamma_{x x}\right\rangle_{\text {ROI }}$ builds up with time in a roughly linear manner [Fig. 2(c)]. The slope of this time evolution varies broadly across cell types and pharmacological interventions. Previous studies established that cellsubstrate stresses depend on substrate stiffness [27,28]. By contrast, we observe that the magnitude and time evolution of $\left\langle\gamma_{x x}\right\rangle_{\text {ROI }}$ are independent of substrate stiffness (Supplemental Material [24] Fig. S8), indicating that monolayer tension is determined by cell-cell adhesion rather than cell-substrate adhesion.

At longer times, monolayers exhibit distinct stress patterns; MDCK cells reach an oscillatory regime, as previously shown, whereas MDCK cells treated with HGF exhibit vanishing tension as the monolayer scatters (Supplemental Material [24] Fig. S6). Similarly, MDCK monolayers lacking $\alpha$-catenin are unable to build up tension, thus, highlighting the collective origin of the observed tension patterns (Supplemental Material [24] Fig. S6).

Our experimental approach provides us with simultaneous yet independent measurements of monolayer tension and strain, thereby allowing us to study the active rheology of the system. Upon plotting tension as a function of strain during the initial buildup phase (Fig. 3), we observe a striking linear relationship and so for all experimental conditions. These results are reminiscent of a rheological tensile test in a purely elastic sheet, where the tension vs strain curve follows a linear dependence whose slope defines an elastic modulus. We note, however, that our experiments probe time scales ranging from $2 \times 10^{4} \mathrm{~s}$ (MDCK-HGF) to $2 \times 10^{5} \mathrm{~s}$ (RPE1), well above the intracellular relaxation time scales [19,29]. Therefore, the apparent elasticity observed in all our experiments must have an active origin. We term it effective active elasticity, and we define the slope of the tension vs strain curve as an active tensile modulus $\left(\Gamma_{x}\right)$ :

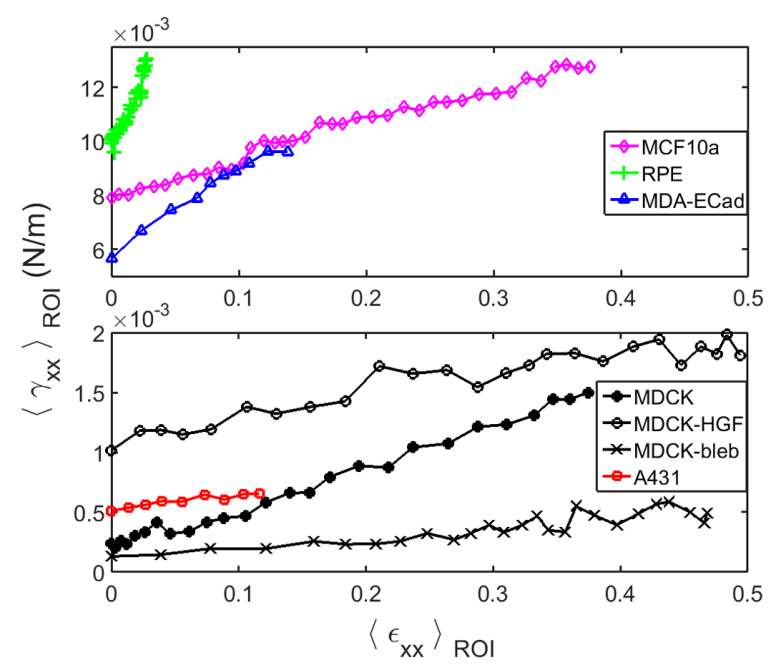

FIG. 3 (color online). Tension-strain relationships for the different experimental conditions. For clarity, the tension axis is split. Same symbols as in Fig. 2. Each curve displays a representative experiment of $n \geq 3$ experiments per condition.

$$
\gamma_{x x}=\Gamma_{x} \epsilon_{x x}+\gamma_{0} .
$$

$\Gamma_{x}$ varies over 2 orders of magnitude across the different cell lines ranging from $\Gamma_{x}=1.30 \pm 0.36 \mathrm{mN} / \mathrm{m}$ for A431 monolayers to $180 \pm 65 \mathrm{mN} / \mathrm{m}$ for RPE1 monolayers (Table I). When myosin activity is inhibited in MDCK monolayers with blebbistatin, $\Gamma_{x}$ decreases sharply, thus, further emphasizing its active origin. Remarkably, the linear behavior spans strains up to 0.4 , a feature that is rarely observed in soft elastic materials, which either strain harden or yield at much lower strains [30-32].

At the beginning of the experiments $\left(\epsilon_{x x}=0\right)$, the monolayer exhibits nonzero tension $\gamma_{0}$, which suggests the existence of a state of vanishing active tension at negative strains, $\epsilon_{0}=-\gamma_{0} / \Gamma_{x}$. By extrapolating the linear relationship in Eq. (2), we estimate the values of such negative strains and the corresponding resting size of each cell type. We interpret this resting size as the one that defines the maximum packing of the monolayer. For MDCK cells, the resting size is $12.1 \pm 1.7 \mu \mathrm{m}$, consistent with the maximum densities reported in the literature for this cell type $[8,23]$. We then define a total cell strain as $\epsilon_{\mathrm{tot}}=\epsilon_{\max }-\epsilon_{0}$, where $\epsilon_{\max }$ is the maximum strain reached by cells during monolayer expansion. $\epsilon_{\text {tot }}$ varies broadly across cell types; while RPE1 monolayers exhibit weak elongation during monolayer expansion $\left(\epsilon_{\text {tot }}=0.069 \pm 0.017\right)$, MDCK cells treated with HGF more than double their resting size $\left(\epsilon_{\text {tot }}=1.37 \pm 0.26\right)$ (Table I).

The active tensile modulus $\Gamma_{x}$ appears to be an intrinsic physical property of each monolayer. We, thus, investigate whether its values can be explained by intrinsic features of the cytoskeleton. As the simplest explanation, we consider the possibility that $\Gamma_{x}$ is proportional to the total amount of myosin that can be activated in the system. When contractility is not pharmacologically inhibited, such 
TABLE I. Values of the main parameters obtained in the study for each experimental condition. For each cell type, the number of repeats is three.

\begin{tabular}{lccccccc}
\hline \hline & MDCK & MDCK-HGF & MDCK-bleb & MCF10a & RPE1 & MDA-Ecad & A431 \\
\hline$\Gamma_{x}(\mathrm{mN} / \mathrm{m})$ & $2.41 \pm 0.41$ & $1.82 \pm 0.32$ & $0.58 \pm 0.26$ & $14.4 \pm 3.2$ & $180 \pm 65$ & $34.7 \pm 5.1$ & $1.30 \pm 0.36$ \\
$\epsilon_{\text {tot }}$ & $0.71 \pm 0.13$ & $1.37 \pm 0.26$ & $0.53 \pm 0.14$ & $0.70 \pm 0.12$ & $0.069 \pm 0.017$ & $0.41 \pm 0.06$ & $0.60 \pm 0.08$ \\
MLC (See Ref. [24], Fig. SI) & $1 \pm 0.15$ & $1.10 \pm 0.12$ & $1.51 \pm 0.25$ & $8.0 \pm 1.6$ & $11.0 \pm 1.9$ & $8.4 \pm 1.8$ & $1.25 \pm 0.16$ \\
$\tau$ (min) & $256 \pm 91$ & $120 \pm 62$ & $195 \pm 19$ & $1180 \pm 280$ & $14100 \pm 2800$ & $1660 \pm 580$ & $903 \pm 428$ \\
\hline \hline
\end{tabular}

amount can be estimated from the total concentration of the myosin light chain (MLC). We use Western blotting [24] to quantify MLC in the different cell types (Table I and Supplemental Material [24] Fig. S9). Upon plotting $\Gamma_{x}$ as a function of MLC, we observe a monotonic dependence (Supplemental Material [24] Fig. S10), thus, supporting that the total myosin that can be phosphorylated is a key determinant of $\Gamma_{x}$. Consistent with this notion, the values of $\Gamma_{x}$ drop significantly when myosin is pharmacologically inhibited (Table I).

We next investigate whether $\epsilon_{\mathrm{tot}}$ can also be explained in terms of intrinsic properties of the cytoskeleton. Following experiments at the single cell level [33], we hypothesize that when the cell reaches its maximum spread size the myosin light chain is maximally phosphorylated. In this case, the maximum active tension $\gamma_{x x_{\max }}$ is

$$
\gamma_{x x_{\max }}=\alpha \mathrm{MLC},
$$

where $\alpha$ is a proportionality factor dependent on myosin energetics and cytoskeletal structure. Replacing this relationship in Eq. (2), we obtain

$$
\Gamma_{x}=\frac{\alpha \mathrm{MLC}}{\epsilon_{\mathrm{tot}}} .
$$

This relationship is consistent with the monolayer data [Fig. 4(a)].

Finally, to further characterize the relationship between monolayer dynamics and myosin activity, we extract the time scale $\tau$ of tissue deformation from a linear fit of the increase of $\epsilon_{x x}(t)$ with time. We find that $\tau$ increases monotonically with the ratio MLC/ $\epsilon_{\text {tot }}$ ([Fig. 4(b)]). This result suggests that the effective viscosity of the monolayer is affected by MLC.

The active tensile modulus reported here defines an active surface tension that links local stress and strain in the monolayer. This quantity is a key input for active models of 2D tissues [34-40], but its value and functional form have been thus far assumed without direct experimental evidence. Using 2D clusters of MDCK cells as a model system, Mertz et al. [11] demonstrated a tissue surface tension defined as the ratio between the total force transmitted to the substrate and the size of the cluster. The surface tension reported by Mertz et al. [11] and the active tensile modulus reported here might share a common origin, but they are defined as the ratio between different physical quantities, and they are obtained in different mechanical scenarios and length scales [see the Supplemental Material [24] Fig. S11).

Our findings reveal a paradox: at ultraslow time scales, monolayers are composed of purely fluid stress-bearing elements, yet they display stress-strain curves characteristic of purely linear solids. A direct implication of this result is that theoretical models of monolayer dynamics should either be based on elastic elements or on an appropriate combination of viscous and active elements that results in effective elastic behavior [41-43].

Recently, Hawkins et al. [44] derived a 1D microscopic model for the remodeling of active solids. At long time scales, the model predicts a linear relationship between stress and strain, thus, defining microscopically an effective elastic modulus. Alternatively, the effective elasticity might originate from an active regulatory mechanism by which cells sense their spread area and regulate their levels of contractility accordingly. How such regulatory mechanism
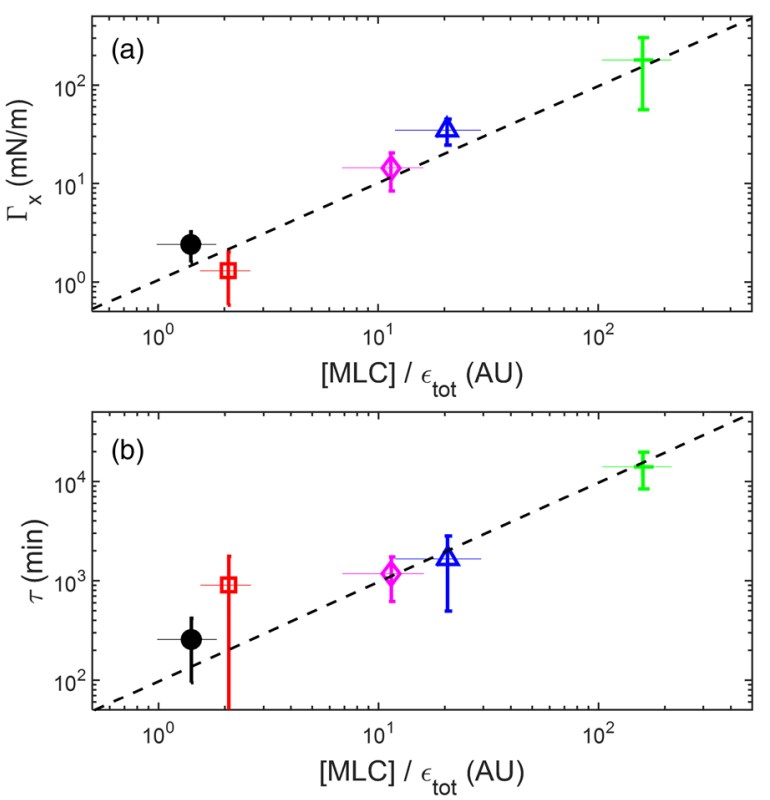

FIG. 4 (color online). (a) $\Gamma_{X}$ increases with $\mathrm{MLC} / \epsilon_{\mathrm{tot}}$. The black line is a power law of exponent 1 , as a guide for the eye. (b) Dependence of the time scale of the remodeling $\tau$ with $\mathrm{MLC} / \epsilon_{\mathrm{tot}}$. The black line is a power law of exponent 1 , as a guide for the eye; same color coding as Fig 2(a). For clarity error bars represent 2 standard deviations. 
might be implemented at the molecular scale and what type of feedback loops are required to maintain linearity up to large strains are major open questions raised by our study.

The authors would like to thank Carles Blanch, Jaume Casademunt, José Muñoz, and Marino Arroyo for discussions and James Nelson, Erik Sahai, David Sarrió, and Gema Moreno-Bueno for contributing cells. This work was supported by the Spanish Ministry of Economy and Competitiveness (Grant No. BFU201238146), the Generalitat de Catalunya (Grant No. 2014SGR-927), and the European Research Council (Grants No. StG-242993 and No. CoG-616480). Carlos Pérez González is supported by Obra Social la Caixa. Marina Uroz is supported by the Spanish Ministry of Economy and Competitiveness (Grant No. BFU2012-38146).

*romaric.vincent@gmail.com †xtrepat@ibecbarcelona.eu

[1] Molecular Biology of the Cell, 4th ed., edited by B. Alberts, A. Johnson, J. Lewis, M. Raff, K. Roberts, and W. Peter, (Garland Science, New York, 2002).

[2] S. Holmes, J. Exp. Zool. 17, 281 (1914).

[3] J. Solon, A. Kaya-Copur, J. Colombelli, and D. Brunner, Cell 137, 1331 (2009).

[4] X. Serra-Picamal, V. Conte, R. Vincent, E. Anon, D. Tambe, E. Bazellières, J. Butler, J. Fredberg, and X. Trepat, Nat. Phys. 8, 628 (2012).

[5] M. Poujade, E. Grasland-Mongrain, A. Hertzog, J. Jouanneau, P. Chavrier, B. Ladoux, A. Buguin, and P. Silberzan, Proc. Natl. Acad. Sci. U.S.A. 104, 15988 (2007).

[6] H. Wensink, J. Dunkel, H. Heidenreich, K. Drescher, R. Goldstein, H. Löwen, and J. Yeomans, Proc. Natl. Acad. Sci. U.S.A. 109, 14308 (2012).

[7] S. Vedula, M. Leong, T. Lai, P. Hersen, A. Kabla, C. Lim, and B. Ladoux, Proc. Natl. Acad. Sci. U.S.A. 109, 12974 (2012).

[8] T. Angelini, E. Hannezo, X. Trepat, M. Marquez, J. Fredberg, and D. Weitz, Proc. Natl. Acad. Sci. U.S.A. 108, 4714 (2011).

[9] K. Nnetu, M. Knorr, J. Kaes, and M. Zink, New J. Phys. 14, 115012 (2012).

[10] J.-A. Park, J. Kim, D. Bi, J. Mitchel, N. Qazvini et al., Nat. Mater. 14, 1040 (2015).

[11] A. F. Mertz, S. Banerjee, Y. Che, G. K. German, Y. Xu, C. Hyland, M. C. Marchetti, V. Horsley, and E. R. Dufresne, Phys. Rev. Lett. 108, 198101 (2012).

[12] D. Tambe, C. Hardin, T. Angelini, K. Rajendran, C. Park, X. Serra-Picamal, E. Zhou, M. Zaman, J. Butler, D. Weitz, J. Fredberg, and X. Trepat, Nat. Mater. 10, 469 (2011).

[13] B. Fabry, G. N. Maksym, J. P. Butler, M. Glogauer, D. Navajas, and J. J. Fredberg, Phys. Rev. Lett. 87, 148102 (2001).

[14] A. Harris, L. Peter, J. Bellis, B. Baum, A. Kabla, and G. Charras, Proc. Natl. Acad. Sci. U.S.A. 109, 16449 (2012).

[15] U. Schwartz and S. Safran, Rev. Mod. Phys. 85, 1327 (2013).

[16] A. Zemel, I. B. Bischofs, and S. A. Safran, Phys. Rev. Lett. 97, 128103 (2006).

[17] A. Lau, B. Hoffman, A. Davis, J. Crocker, and T. Lubensky, Phys. Rev. Lett. 91, 198101 (2003).
[18] P. Bursac, G. Lenormand, B. Fabry, M. Oliver, D. Weitz, V. Viasnoff, J. Butler, and J. Fredberg, Nat. Mater. 4, 557 (2005).

[19] B. Hoffman, G. Massiera, K. Van Citters, and J. Crocker, Proc. Natl. Acad. Sci. U.S.A. 103, 10259 (2006).

[20] D. Mizuno, C. Tardin, C. Schmidt, and F. MacKintosh, Science 315, 370 (2007)

[21] D. Riveline, E. Zamir, N. Balaban, U. Schwarz, T. Ishizaki, S. Narumiya, Z. Kam, B. Geiger, and A. Bershadsky, J. Cell Sci. 153, 1175 (2001).

[22] S. Dupont, L. Morsut, M. Aragona, E. Enzo, S. Giulitti, M. Cordenonsi, F. Zanconato, J. Le Digabel, M. Forcato, S. Bicciato, N. Elvassore, and S. Piccolo, Nature (London) 474, 179 (2011).

[23] X. Trepat, M. Wasserman, T. Angelini, E. Millet, D. Weitz, J. Butler, and J. Fredberg, Nat. Phys. 5, 426 (2009).

[24] See the Supplemental Material at http://link.aps.org/ supplemental/10.1103/PhysRevLett.115.248103, which includes Refs. [25,26], for full details of the experimental methods, and additional figures referenced in the Letter.

[25] D. Sarrió, J. Palacios, M. Hergueta-Redondo, G. GómezLópez, A. Cano, and G. Moreno-Bueno, BMC Cancer 9, 74 (2009).

[26] R. Li and Y. Shen, Life Sci. 92, 747 (2013).

[27] C. E. Chan and D. J. Odde, Science 322, 1687 (2008).

[28] A. Elosegui-Artola, E. Bazellières, M. D. Allen, I. Andreu, R. Oria, R. Sunyer, J. J. Gomm, J. F. Marshall, J. L. Jones, X. Trepat, and P. Roca-Cusachs, Nat. Mater. 13, 631 (2014).

[29] P. Marmottant, A. Mgharbel, J. Käfer, B. Audren, J.-P. Rieu, J.-C. Vial, B. van der Sanden, A. Marée, F. Graner, and H. Delanoë-Ayari, Proc. Natl. Acad. Sci. U.S.A. 106, 17271 (2009).

[30] M. Gardel, J. Shin, F. MacKintosh, L. Mahadevan, P. Matsudaira, and D. Weitz, Science 304, 1301 (2004).

[31] C. Storm, J. Pastore, F. MacKintosh, T. Lubensky, and P. Jamney, Nature (London) 435, 191 (2005).

[32] T. G. Mason and D. A. Weitz, Phys. Rev. Lett. 75, 2770 (1995).

[33] T. Polte, G. Eichler, N. Wang, and D. Ingber, Am. J. Physiol., Cell Physiol. 286, C518 (2004).

[34] J. Zimmermann, R. Hayes, M. Basan, J. Onuchic, W.-J. Rappel, and H. Levine, Biophys. J. 107, 548 (2014).

[35] A. Brugués, E. Anon, V. Conte, J. Veldhuis, M. Gupta, J. Colombelli, J. Muñoz, G. W. Brodland, B. Ladoux, and X. Trepat, Nat. Phys. 10, 683 (2014).

[36] E. Hannezo, J. Prost, and J.-F. Joanny, Proc. Natl. Acad. Sci. U.S.A. 111, 27 (2014).

[37] S. Banerjee, K. J. C. Utuje, and M. C. Marchetti, Phys. Rev. Lett. 114, 228101 (2015).

[38] J. Arciero, Q. Mi, M. Branca, D. Hackam, and D. Swigon, Biophys. J. 100, 535 (2011).

[39] P. Lee and C. Wolgemuth, PLoS Comput. Biol. 7, e1002007 (2011).

[40] M. Köpf and L. Pismen, Soft Matter 9, 3727 (2013).

[41] F. C. MacKintosh and A. J. Levine, Phys. Rev. Lett. 100, 018104 (2008).

[42] T. Liverpool, M. Marchetti, J.-F. Joanny, and J. Prost, Europhys. Lett. 85, 18007 (2009).

[43] B. Shiladitya and M. Marchetti, Soft Matter 7, 463 (2011).

[44] R. J. Hawkins and T. B. Liverpool, Phys. Rev. Lett. 113, 028102 (2014). 